\title{
Highlights from the San Antonio Breast Cancer Symposium 2011
}

\author{
Chair: Georg Pfeiler \\ Participants: Peter Dubsky ${ }^{b}$ Günther G. Steger ${ }^{c}$ Gunter von Minckwitz ${ }^{d}$ \\ Beat Thürlimann ${ }^{e}$ Michael Untch ${ }^{f}$

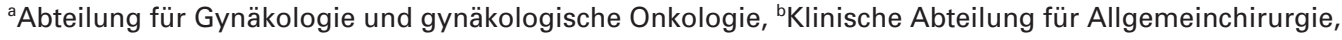 \\ ${ }^{c}$ Klinische Abteilung für Onkologie, Medizinische Universität Wien, Austria, ${ }^{d} G B G$ Forschungs GmbH, Neu-Isenburg, Germany \\ ${ }^{\mathrm{e} B r u s t z e n t r u m, ~ K a n t o n s s p i t a l ~ S t . G a l l e n, ~ S w i t z e r l a n d, ~}{ }^{\mathrm{f}}$ Brustzentrum, HELIOS-Klinikum Berlin-Buch, Germany
}

\section{Question 1: How Do the New Results on Bisphospohnates Change Your Thinking and Daily Clinical Practice When Dealing With Adjuvant Therapy of Premenopausal and Postmenopausal Patients With Breast Cancer?}

Dubsky: I am a co-author of the ABCSG 12 study and have been working very close to these data for several years. It has been quite remarkable to witness how well the data have matured, the hazard ratios (HRs) have kept stable and it is now of course a further credit to the study to show a significant overall survival advantage. In Austria, 3 years of goserelin in combination with tamoxifen has been a standard endocrine therapy for most oncologists ever since publication of the ABCSG 5 results and the start of ABCSG 12 over a decade ago. Currently, we are using zoledronic acid (ZOL) $4 \mathrm{mg}$ every 6 months for 3 years to treat women that fit the inclusion criteria of ABCSG 12. This means that estrogen receptor positive $(\mathrm{ER}+)$ premenopausal patients not assigned to chemotherapy receive adjuvant $\mathrm{ZOL}$ on the basis of the ABCSG 12 data and its publication. We are well aware that this schedule is not a standard therapy in other parts of the world - and this makes the adjuvant use of ZOL limited. Concerning the use of ZOL in postmenopausal women there are currently insufficient data to recommend its use as an adjuvant therapy. Although the subset analysis of AZURE showing both disease free survival (DFS) and overall survival (OS) advantages for postmenopausal women is very impressive (and comprises the largest group of women that we treat!), the overall study is negative. However, especially postmenopausal women treated with aromatase inhibitors (AI) show high rates of bone loss; several guidelines recommend the use of bisphosphonates in this indication. von Minckwitz: With two further negative trials and subgroup second endpoint analysis from 2 others I'm much more skeptical about using bisphosphonates now. The ABCSG study is not transferable to our current treatment strategies.

Steger: ABCSG 12 has now demonstrated a significant survival benefit in premenopausal patients with hormone sensitive tumors without showing any long-term toxicity concerns and especially no risk for osteonecrosis of the jaw (ONJ). These results were discussed by James Ingle (Mayo Clinic) who concluded that adjuvant zoledronic acid as used in the Austrian study is a new standard of care. Thus, I think it is justified to offer this adjuvant treatment with zoledronic acid to patients with the same characteristics as the ABCSG 12 study population. Since a subgroup analysis of the AZURE trial in postmenopausal patients with hormone-sensitive tumors also showed a benefit for the truly postmenopausal women, these patients should be considered for adjuvant zoledronic acid and the pros and cons should be discussed with the individual patient.

Thürlimann: Based on subgroup and partly retrospective analyses, we see a signal for possible useful clinical activity of bisphosphonates in postmenopausal women. However, the two most powerful trials to investigate the use of bisphosphonates were clearly negative for the primary endpoint (AZURE, NSABP B-34). Thus, we have not adopted the routine use of bisphosphonates as an antineoplastic adjuvant therapy in daily practice for both pre- and postmenopausal patients. It is reassuring to know that bisphosphonates given for preservation of bone integrity might also benefit the patients at risk for relapse.

\section{KARGER \\ Fax +497614520714 \\ Information@Karger.de}

www.karger.com (c) 2012 S. Karger GmbH, Freiburg

$1661-3791 / 12 / 0071-0068 \$ 38.00 / 0$

Accessible online at:

www.karger.com/brc ass. Prof. Dr. Georg Pfeiler

Abteilung für Gynäkologie und gynäkologische Onkologie

MUW, AKH Wien

Währinger Gürtel 18-20, 1090 Wien, Austria

Tel. +43 140400 2801, Fax -2323

georg.pfeiler@meduniwien.ac.at 
Untch: One of the first papers with the use of the bisphosphonate clodronate in a prevention trial was published by Ingo Diel [New Engl J Med 1998;6:357-363]. A positive effect of adjuvant clodronate in terms of recurrence and metastasis was shown in the treatment of breast cancer patients. A second paper was published by Michael Gnant et al. [New Engl J Med 2009;360:679-691], showing a positive effect of zoledronic acid as adjuvant preventive treatment in patients with primary breast cancer. In a trial published by Robert Coleman [Coleman et al., N Engl J Med 2011;365:1396-1405] more than 3,000 patients were randomized to receive standard treatment with surgery, radiotherapy, chemotherapy and endocrine therapy. The patients were randomized to either control or zoledronic acid in a $4 \mathrm{mg}$ dose by intravenous infusion at each cycle of chemotherapy and then every 3 months for 8 doses, followed by 5 cycles on every 6 months scheduled for a total of 5 years. DFS was not different between the study arms. The subgroup of patients who were more than 5 years after menopause showed a significant benefit from the addition of zoledronic acid with a relative risk reduction of $25 \%$. The incidence of ONJ was $1.1 \%$ in the zoledronic acid arm. The positive effect from the subgroup analysis led to the recommendation of adjuvant therapy with zoledronic acid by the National German Guideline Commission of the AGO.

At the San Antonio Breast Cancer Meeting in December 2011 Michael Gnant presented the results of the ABCSG 12 trial. 1,803 premenopausal patients were randomized to receive either endocrine treatment with tamoxifen or anastrozole with goserelin or the same endocrine treatment plus $4 \mathrm{mg}$ zoledronic acid every 6 months for 3 years. The study endpoint of this trial was achieved with a significantly better DFS in the zoledronic acid study arm, with a relative risk reduction of $29 \%$. Patients under the age of 40 had no benefit from this therapy; a significant benefit from this therapy was seen in patients older than 40 , with a relative risk reduction of $33 \%$. On the other hand, the German adjuvant intergroup node positive study (GAIN) presented by Volker Möbus at the San Antonio Breast Cancer Meeting 2011 has shown no significant benefit from the addition of 2 years of $50 \mathrm{mg}$ daily ibandronate. DFS and OS were identical with or without the addition of the bisphosphonate. Subgroup analyses in the Forrest plot have shown a trend towards an effect of the bisphosphonate in patients older than 60 years. The study population of this trial was mainly composed of high-risk and younger patients. One possible explanation from this trial is that either compliance with the oral medication was not good enough or the oral resorption of this medication might not be optimal. Mainly, the benefit of bisphosphonates in the adjuvant setting has been in patients with an increased bone turnover either by the addition of GnRH analogue in the premenopause or in patients older than 60 or 65 years. Another trial, the ZOFAST study was presented at the San Antonio Breast Cancer Meeting 2011. More than 1,000 patients were randomized either to letrozole plus the immediate addition of zoledronic acid, 4 mg every 6 months or letrozole and delayed zoledronic acid for a total treatment duration of 5 years. There was a significant increase in DFS in the immediate zoledronic acid study arm with a relative risk reduction of $33 \%$.

In summary, we have several positive trials with i.v. bisphosphonates like ZO-FAST, AZURE, and ABCSG 12 with a hazard ratio between 0.6 and 0.7 by the addition of zoledronic acid. In my opinion, there is a strong evidence to recommend i.v. bisphosphonates, such as $4 \mathrm{mg}$ zoledronic acid every 6 months to patients with postmenopausal breast cancer in the adjuvant setting.

\section{Question 2: According to Results of the GeparTrio Trial, Intermediate Adaption of Neoadjuvant Chemotherapy May Improve Disease Outcome. Do You Think That this Leads to a Further Indication of Neoadjuvant Chemotherapy? How Do You Deal With Non-Responders after 2-4 Cycles of Neoadjuvant Chemotherapy?}

Dubsky: The German Breast Group (GBG) presented the secondary outcomes of their GeparTrio trial. The investigators found that response guided treatment at 5 years of follow-up increased both DFS and OS. The effect was primarily driven by luminal type tumors - not by triple negative breast cancer (TNBC) or ER-/Her2+ breast cancer. The GBG has previously shown that $\mathrm{pCR}$ in luminal tumors does not have a prognostic effect (ASCO 2011). What are the clinical implications? This study is very difficult to translate into practice at this stage. TAC (docetaxel, doxorubicin and cyclophosphamide) is a toxic regimen - and today few oncologists would recommend giving a full 8 cycles. Furthermore, in the nonresponding part of the population it is not clear if the results from neoadjuvant chemotherapy had been similar if a sequential preoperative regimen such as EC (epirubicin and cyclophosphamide) followed by $\mathrm{T}$ (docetaxel) had been used. The data currently raise more questions than they are able to answer for current clinical routine. For clinical research this study will change the preoperative trial landscape. There are several possibilities to treat non responding women with less toxic regimens over an extended period of time - this would improve quality of life and may at the same time lead to clearcut survival benefits.

In my opinion, preoperative chemotherapy, other than improving breast conservation rates, in the beginning had two major goals. One was to improve survival by adding systemic treatment before surgery - this goal has not been accomplished, although many would suggest that this might be true for a subset of young women with aggressive tumors. The second goal was to use the in vivo sensitivity testing for treatment decisions; again, this was aimed at improving both breast conservation and survival. The secondary outcome of GeparTrio has raised hopes that we may be able to accom- 
plish this for our patients. Unfortunately, several clinical trials (with 'old fashioned' survival endpoints) will be needed to move this idea into clinical practice. I look forward to read these data in full manuscript and would like to congratulate GBG for this mind changing study.

von Minckwitz: With my bias as primary investigator, I believe it will push neoadjuvant chemotherapy forward. As we use a lot of sequential treatment we switch anyhow. However, it might be another argument to use the taxane first.

Steger: I don't think that these results will lead to a further indication of neoadjuvant chemotherapy but the data show very clearly that the clinical and/or radiological response evaluation is crucial and that early adaption of the treatment schedule if response is suboptimal benefits many patients. These results from a prospective trial now support the clinical practice where this has already been done in the past outside clinical trials, e.g. when the patients' desire for breast conservation was paramount and the first-line treatment was insufficient to downstage the primary tumor. Furthermore, this possibility to individualize the treatment according to the individual response is one of biggest advantages of neoadjuvant therapy.

Thürlimann: No, I don't think that these results might lead to further indications. The definite answer to the question of how to treat non-responders remains unclear as several other trials did not show the same findings as GeparTrio. A change of regimen in non-responders is indeed an option as is to go directly to surgery in large operable tumors. This must be decided case by case.

Untch: The GeparTrio trial is a landmark neoadjuvant trial, which was started almost 10 years ago and published for the first time in the Journal of the National Cancer Institute 2008. The long-term follow-up of this study will be practice changing. After 2 cycles of neoadjuvant chemotherapy with TAC patients were randomized: patients with a complete or partial response assessed by palpation or sonography received either 4 additional cycles of TAC or 6 additional cycles of TAC. The patients with no change after 2 TAC cycles received either an alternative chemotherapy with capecitabine and navelbine or 4 cycles of TAC. DFS and OS were significantly better in patients who had a response guided treatment compared to the conventional treatment. Response guided treatment reduced the relative risk of recurrence and metastases by $29 \%$ and the risk of death by $21 \%$. In patients who had a good response after 2 cycles of TAC, those patients with an additional 6 cycles (total 8 cycles) had a significantly better outcome compared to patients with additional 4 cycles TAC (total number of cycles 6). The relative risk reduction was $22 \%$. In non-responding patients the switch to another chemotherapy (navelbine plus capecitabine) increased the DFS compared to the conventional treatment (e.g., continu- ing TAC for a total number of 6 cycles) with a relative risk reduction of $61 \%$. Luminal B HER2- patients had a significant benefit from the therapy. The response guided treatment was significantly better than conventional treatment. The downside was shown in patients with triple negative tumors. Patients with a pathologic complete response (pCR) had an excellent outcome. On the other hand, patients with triple negative tumors and no pCR had an almost 7 times higher rate of recurrence and metastases. In patients with triple negative tumors the response guided therapy after 2 cycles of TAC had no significant benefit on the outcome. A similar situation was seen in patients with HER2+ non-luminal tumors: those patients who had a pCR had a significant benefit in terms of outcome. On the other hand, the response guided treatment after 2 cycles TAC had no significant benefit on outcome. This was before trastuzumab was used in the neoadjuvant therapy of patients with HER2 overexpressing tumors. In a second presentation by Sibylle Loibl at the San Antonio Breast Cancer Meeting 2011 interesting results were shown from a metaanalysis with more than 6,000 patients who were analyzed for pCR, DFS, and OS according to different tumor subtypes. From the metaanalysis of neoadjuvant trials with the addition of trastuzumab we know that patients with a pCR have an excellent outcome and patients without pCR have a significant rate of recurrence and metastasis. These patients need alternative anti-HER2 treatment (postneoadjuvant). Such trials are planned. In the meantime, we recruit patients in the GeparSixto trial in which patients with HER2+ disease are treated with anthracycline, taxane, and the addition of double blockade with trastuzumab and lapatinib. The GeparSepto trial which is going to start this year will randomize more than 1,200 patients. Patients with HER2 overexpressing tumors will be treated with a double blockade with trastuzumab and pertuzumab.

\section{Question 3: Which Risk Assessment Tool Do You Use/ Would You Like to Use Additionally to the Common Histopathological and Clinical Criteria? Do We Need Them All?}

Dubsky: At the Medical University of Vienna (MUV) we are currently using the Mammaprint ${ }^{\circledR}$ test (Agendia BV, Amsterdam, The Netherlands) in a decision-making study in ER+/ Her2- women that we consider to have low to intermediate risk. In these patients the chemo/endocrine question is usually difficult to answer. We find that the additional prognostic information is helpful in select patients. Together with the Austrian Breast and Colorectal Cancer Group (ABCSG) we have furthermore validated the Endopredict test [Filipits et al., Clin Cancer Res 2011;17:6012-6020], a combined molecular and clinical test (EPclin). Using the EPclin we are very confident in identifying a group of ER+ postmenopausal women that are treated with endocrine therapy in the absence of 
chemotherapy that have a remarkably low rate of distant metastases at 10 years of follow-up. During the SABCS 2011 meeting the ROR46 (risk of recurrence 46 genes) test has been presented. The ROR is a molecular tool that also includes tumor size and is derived from the PAM50 molecular subsetting. The ROR was validated in the transATAC cohort and the prognostic likelihood of distant metastases was compared to the Recurrence Score (RS; Genomic Health) and the IHC4. The ROR compared favorably to the RS and seemed comparable to the IHC4 score. Concerning the daily clinical implication of this test, I am however unable to draw any conclusions. In the absence of prospective data none of these tests should be used to predict benefit from chemotherapy. The goal is always to identify women with a very low risk; or a risk deemed low in view of competitive health risks in order to improve decision-making. The comparison of prognostic tests, no matter what statistical measures and endpoints are used, is not clinically helpful. At this point clinicians should consider the following criteria when choosing molecular tools for their ER+/Her2- patients:

a) The test should be robust and validated in patient cohorts with the highest possible quality of clinical data (i.e., from phase III clinical trials).

b) The test should clearly add information to the clinicopathologic variables.

c) The test should be more sensitive in identifying patients with a very low risk than any algorithm of clinical and pathological parameters would allow.

d) The test should be feasible in terms of shipping, cost, tissue requirements, and time.

von Minckwitz: I will explore more PAM50 as a potential tool.

Steger: Since the results of the appropriate prospective trials TAILORx and MINDACT are still not available, the use of genetic assays should not be used outside a trial scenario. For the time being, the knowledge of the 4 receptors, i.e. ER, PR, HER2, and Ki67 should suffice for appropriate selection of therapy.

Thürlimann: There are no new results which encourage us today to use other assessment tools of prognostic factors (e.g., Oncotype DX ${ }^{\circledR}$, Genomic Health Deutschland GmbH, Munich, Germany). Therefore, we follow the 2011 St. Gallen Consensus recommendations and use conventional pathology tests with internal and external quality control. Results from the big MINDACT and TAILORx trials will hopefully add useful information on the additional value of such tests.

Untch: Risk assessment tools are needed especially in patients with hormone receptor positive disease with G1, G2 tumors and uninvolved axillary lymph nodes or patients with 1-3 positive axillary nodes. At the moment, the German National Guideline recommends the routine use of uPA/PAI 1. We use these markers in our Breast Cancer Center and also at all HELIOS Breast Cancer Centers in Germany. Additionally, the immunohistochemical detection of $\mathrm{Ki} 67$ is a routine test for the classification of hormone receptor positive tumors in luminal A and luminal B tumors. The use of the Recurrence Score ${ }^{\circledR}$ (Genomic Health) is limited in Germany and not recommended by our National Guideline Commission. Some new tests like PAM50 and EndoPredict from Sividon (Sividon Diagnostics $\mathrm{GmbH}$, Cologne, Germany) are very promising, especially because they can be performed by the local pathologist, but we still need additional data from studies.

\section{Question 4: Will the m-Tor Inhibitor Be Our Next Targeted (?) Therapy in Breast Cancer?}

Dubsky: Everolimus has been developed in ER+ recurrent breast cancer. TAMRAD and BOLERO-2, two studies in recurrent breast cancer have shown remarkable results: whereas in the much smaller TAMRAD study $(\mathrm{n}=111)$ the subset analysis suggests treatment effects being driven by patients with secondary resistance to endocrine therapy, the BOLERO-2 study ( $\mathrm{n}=724)$ showed excellent benefit regardless of prior sensitivity to endocrine treatment. The increase in median PFS in BOLERO-2 was almost 7 months, the HRs in both studies are well below 0.5 indicating a $50 \%$ risk reduction - these data may lead to a bona fide OS benefit in the future. Class specific toxicities include stomatitis and rare cases of pneumonitis, both of which seemed to be taken care of with dose reductions or short therapy breaks. This is an interesting option to keep ER+ advanced patients away from chemotherapy with good quality of life and excellent long-term survival results. In the future, inhibiting the mammalian target of Rapamycin (m-TOR) pathway may be an excellent option for $\mathrm{ER}+$ patients at higher risk - even in the adjuvant setting. Efforts are ongoing to move this drug into the adjuvant setting.

von Minckwitz: Yes. The effect is very intriguing. Adjuvant studies are urgently needed.

Steger: The astonishing results of the BOLERO-2 trial showing a hazard ratio of 0.36 for the PFS and early signs for a possible OS advantage when a m-TOR inhibitor is added to an aromatase blocker in postmenopausal women with hormone dependent disease are more than promising. I am sure that these results will soon lead to a rapid approval of everolimus in this indication. However, since we are lacking a measurable target for proper patient selection, the requirements to be called a targeted therapy are not fulfilled.

Thürlimann: Indeed, BOLERO-2 and TAMRAD are milestones in the history of endocrine treatment of breast cancer. We have not only learnt a lot about mechanisms of endocrine resistance but have now robust data showing clinically feasible 
and efficient treatment. However, the side-effects and costs must be considered.

Untch: The m-TOR molecule has come into our focus especially by the presentation of the TAMRAD study in San Antonio 2010 and by the exceptionally positive data from the BOLERO-2 trial presented by G. Hortobagy at the San Antonio Breast Cancer Meeting 2011. One of the most challenging topics in the treatment of patients with metastatic breast cancer is the development of resistance to hormonal therapy and the shift to tumorigenic signaling through alternative pathways. This is mainly driven by the m-TOR molecule. The blockade of m-TOR by everolimus (RAD-001) was the most exciting presentation from San Antonio 2011. Patients with multiple previous treatments for metastatic disease with $\mathrm{HR}+$ tumors were treated either with exemestane or with exemestane plus everolimus. More than 700 patients were entered into this trial. The relative risk reduction for progression was $60 \%$ by the addition of everolimus, with a $\mathrm{p}$ value of $10^{-16}$. From the clinical point of view we would like to use this combination as soon as possible for our patients with hormone receptor-positive metastatic disease.

\section{Question 5: In Your Oppinion, What Are the Most Relevant Clinical Proceedings Regarding HER2-Positive Breast Cancer?}

Dubsky: HER2+ breast cancer is an intriguing field and moving quickly on many fronts. Several abstracts on findings in both mouse and human indicate that HER2 overexpressing patients as defined by ASCO/CAP may not be the only patients benefiting from therapies targeted at the receptor. However, clinical trials to move this into clinical practice are still ongoing. Furthermore, clinicians are also much more sensitive about differentiating between ER+/HER2+ patients and non-luminal HER2+ women. There is an increasing interest in the possibility to treat the ER+ subset without chemotherapy. Finally, there is half a dozen of new agents targeted at HER2 and description of their current development goes well beyond the scope of this expert discussion. Pertuzumab is a fully humanized monoclonal antibody that inhibits ligand dependent dimerization of HER2. Furthermore, and much like trastuzumab it may gain additional potency by activating ADCC. Data from CLEOPATRA, a large phase III study in untreated HER2+ metastatic breast cancer patients, and response rates and toxicity from TRYPHAENA, a preoperative phase II chemotherapy trial, certainly add momentum to the development of pertuzumab. Pertuzumab will advance as an additional treatment option in metastatic disease and has shown excellent efficacy in preoperative phase II trails. The adjuvant development is ongoing with a very large multinational trial now recruiting patients in APHINITY (BIG 4-11/ BO25126/TOC4939g). von Minckwitz: The strong effect of pertuzumab. Adjuvant data will be very interesting.

Steger: The development of further anti-HER2-agents like pertuzumab and trastuzumab-emtansine (TDM-1) which are already in the clinic and which will be available in due course. Moreover, the data from the recent neoadjuvant trials in HER2-positive disease showing remarkable activity when anti-HER 2 compounds are combined and this also even in the chemotherapy-free treatment arms are very promising for the near future.

Thürlimann: The most important developments are the introduction of double HER2 blockade by trastuzumab and pertuzumab. This offers a clinically useful treatment option even without chemotherapy. Furthermore, T-DM1 showed promising results and is both a milestone in and a model for the entire field of oncology. The optimal use of these drugs will now soon be investigated in the adjuvant setting (APHINITY study) and in the palliative setting (by SAKK and UNICANCER).

Untch: In patients with HER2-positive disease, one of the most exciting abstracts was the presentation of the CLEOPATRA trial by Jose Baselga. In this trial more than 800 patients were randomized between the standard treatment taxane and trastuzumab versus taxane and trastuzumab plus the addition of the antibody pertuzumab. The relative risk reduction in terms of disease progression was almost $40 \%$ and the relative risk reduction in terms of OS was more than $33 \%$ by pertuzumab. This trial will be definitively practice changing in patients with HER2-positive disease and also very promising adjuvant (APHINITY) and neoadjuvant trials with the double blockade trastuzumab and pertuzumab (GeparSepto) will be started soon.

\section{Question 6: Is Axillary Lymph Node Dissection Following a Positive SLN ( $<2 \mathrm{~mm},>2 \mathrm{~mm}$ ) Standard of Care?}

Dubsky: In our own practice we divide the question into two separate issues.

a) The minimally involved (single) sentinel lymph node $(S L N)$ micrometastasis $(<2 \mathrm{~mm})$ : although most likely of prognostic significance is unlikely to have an influence on survival in the context of adjuvant therapy. We therefore do not perform axillary clearance unless there is no possibility of adjuvant therapy or several other risk factors are present, including multiple micrometastases, several involved sentinel lymph nodes or aggressive tumor biology.

b) SLN macrometastasis (>2 $\mathrm{mm}$ ) has led to conflicting opinions and emotional discussions in our environment. Several prior lines of evidence suggest that not every lymph 
node with metastatic spread leads to axillary recurrence and much less to decreased survival. This year's publication of the ACOSOG Z011 has very much added to this idea. However, even if the strict inclusion criteria of this study are applied (cT1/2; cN0, etc.) many are reluctant to forgo axillary dissection (AD). Indeed, there are many issues that the ACOSOG Z011 should address. What exactly were the radiotherapy protocols? How likely does it seem that patients did not receive $\mathrm{AD}$ but radiotherapy of levels I and II? Is this trial practice changing or just power challenged? Furthermore, most clinicians cannot seem to identify the amount of morbidity of AD published in their own patients. ACOSOG Z011 is an important trial and like all important trials leaves many questions unanswered. It is also clear that a repeat trial is not feasible. In Austria we are currently developing a prospective registry for women that have not undergone $\mathrm{AD}$ in the face of positive sentinel nodes. In the meantime interdisciplinary team management should be reminded that the issue is limited to '...women who have clinical T1-T2 breast cancer and hematoxylin-eosin-detected metastasis in the SLN and who are treated with breast-conserving surgery, whole-breast irradiation, and adjuvant systemic therapy' [Giuliano et al. JAMA 2011;305:569-575]. We find that on the ground, meaning in daily practice, the decision-making process is quite straightforward.

von Minckwitz: Forgoing axillary dissection even with a positive SLN should be considered more frequently (maybe also depending on subtype).

Steger: I don't want to fuel a meanwhile endless and not always fruitful surgical discussion on what the 'Standard of Care' (SOC) is in these situations. From an interdisciplinary oncologic point of view I advocate an adequate discussion within each breast cancer unit, where all recent trial results should be considered when the institutions SOC is defined. However, also the limitations of these trials as well as other risk factors for local and/or systemic relapse must be taken into consideration to define the institution's SOC in order to benefit the patients best.

Thürlimann: As we learned from the ACOSOG Z0011 trial during last ASCO, axillary dissection is frequently unnecessary despite positive findings in the SLN. Based on these results and findings of other trials, we adopted the omission of axillary clearance for patients in the specific setting of the Z0011 trial. Thus we fully endorse the St. Gallen Consensus statement and do not extrapolate these findings to other patients.

Untch: The omission of axillary lymph node dissection following a positive sentinel lymph node is a standard of care according to our German guideline recommendation of the AGO from 2011. This recommendation is based on the
ACOSOG Z0011 study from Armando Giuliano who received the San Antonio Breast Cancer Merit Award in the Year 2011 for this practice changing study. This has been also recommended by the St. Gallen Conference in the year 2011, the proceedings are published in the Annals of Oncology 2011. Patients with invasive breast cancer with less than $5 \mathrm{~cm}$ tumor diameter with clinically and sonographically unsuspicious lymph nodes in the ipsilateral axilla who are treated with breast conserving therapy and tangential field irradiation do not need additional AD if 1 or 2 SLN are involved with micro- or macrometastases. If 3 or more SLN are involved, $\mathrm{AD}$ still has to be performed. Patients with a mastectomy are also candidates for an AD if 1 or 2 SLN are involved. Patients with extracapsular involvement outside of the SLN are also candidates for additional AD. This statement was extensively discussed at the San Antonio Breast Cancer Meeting 2011, and surgeons worldwide are about to embark on this new approach which is another step in the reduction of side effects caused by breast cancer surgery.

\section{Participants}

Priv. Doz. Dr. Peter Dubsky

Klinische Abteilung für Allgemeinchirurgie

Medizinische Universität Wien

Währinger Gürtel 18-20

1090 Wien, Austria

Tel. +43 1 40400-6916, Fax -6918

peter.dubsky@meduniwien.ac.at

Univ.-Prof. Dr. Günther G. Steger

Klinische Abteilung für Onkologie

Medizinische Universität Wien

Währinger Gürtel 18-20

1090 Wien, Austria

Tel. +43 1 40400-5359, Fax -6081

guenther.steger@meduniwien.ac.at

Prof. Dr. med. Gunter von Minckwitz

GBG Forschungs $\mathrm{GmbH}$

Martin Behaim Strasse 12

63263 Neu-Isenburg

Tel. +49 6102 7480-411, Fax -111

Gunter.vonMinckwitz@germanbreastgroup.de

Prof. Dr. med. Beat Thürlimann

Brustzentrum

Kantonsspital St. Gallen

9007 St. Gallen, Switzerland

Tel. +41 71 494-18 88, Fax -63 68

beat.thuerlimann@kssg.ch

Prof. Dr. med. Michael Untch

Brustzentrum

HELIOS-Klinikum Berlin-Buch

Schwanebecker Chausse 50

13125 Berlin, Germany

Tel. +49 30 9401 53-301, Fax -309

muntch@berlin.helios-kliniken.de 